\title{
Moisture uptake in nanocellulose: the effects of relative humidity, temperature and degree of crystallinity
}

\author{
Mohit Garg • Varvara Apostolopoulou-Kalkavoura • Mathieu Linares • \\ Tahani Kaldéus • Eva Malmström • Lennart Bergström • Igor Zozoulenko
}

Received: 20 January 2021 / Accepted: 18 July 2021 / Published online: 29 July 2021

(C) The Author(s) 2021

\begin{abstract}
Foams made from cellulose nanomaterials are highly porous and possess excellent mechanical and thermal insulation properties. However, the moisture uptake and hygroscopic properties of these materials need to be better understood for their use in biomedical and bioelectronics applications, in humidity sensing and thermal insulation. In this work, we present a combination of hybrid Grand Canonical Monte Carlo and Molecular Dynamics simulations and experimental measurements to investigate the moisture uptake within nanocellulose foams. To explore the effect of surface modification on moisture uptake we used two types of celluloses, namely TEMPO-oxidized cellulose nanofibrils and
\end{abstract}

Mohit Garg and Varvara Apostolopoulou-Kalkavoura have contributed equally.

Supplementary Information The online version contains supplementary material available at https://doi.org/10.1007/ s10570-021-04099-9.

M. Garg · M. Linares · I. Zozoulenko $(\bowtie)$

Laboratory of Organic Electronics, Department of Science and Technology (ITN), Linköping University, Campus

Norrköping, 60174 Norrköping, Sweden

e-mail: igor.zozoulenko@liu.se

V. Apostolopoulou-Kalkavoura · L. Bergström ( $\square)$

Department of Materials and Environmental Chemistry, Stockholm University, Svante Arrhenius väg 16C,

10691 Stockholm, Sweden

e-mail: lennart.bergstrom@mmk.su.se carboxymethylated cellulose nanofibrils. We find that the moisture uptake in both the cellulose nanomaterials increases with increasing relative humidity $(\mathrm{RH})$ and decreases with increasing temperature, which is explained using the basic thermodynamic principles. The measured and calculated moisture uptake in amorphous cellulose (for a given $\mathrm{RH}$ or temperature) is higher as compared to crystalline cellulose with TEMPO- and CM-modified surfaces. The high water uptake of amorphous cellulose films is related to the formation of water-filled pores with increasing $\mathrm{RH}$. The microscopic insight of water uptake in nanocellulose provided in this study can assist the design and fabrication of high-performance cellulose materials with improved properties for thermal insulation in humid climates or packaging of water sensitive goods.

\author{
M. Linares \\ Group of Scientific Visualization, Department of Science \\ and Technology (ITN), Linköping University, Campus \\ Norrköping, 60174 Norrköping, Sweden \\ M. Linares \\ Swedish E-Science Center (SeRC), Linköping University, \\ 58183 Linköping, Sweden
}




\section{Graphic abstract}

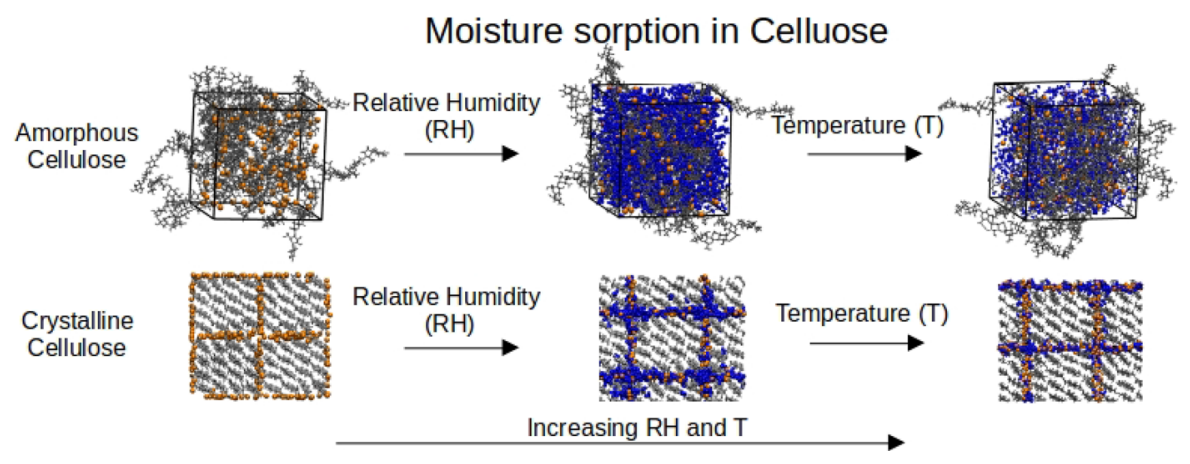

Keywords Cellulose - Relative humidity - Foams · Grand Canonical Monte Carlo · Molecular dynamics simulations

\section{Introduction}

Cellulose is one of the most abundant biopolymers on Earth. It can be found in wood, algae, tunicates, and cotton usually combined with other components such as hemicellulose and lignin (Cosgrove 2005). Cellulose is a light-weight and versatile material that exhibits a structural hierarchy from the ångstrom- to the micrometer scale, and cellulose nanomaterials (CNMs) display a high surface area and tunable surface chemistry making them versatile materials for a variety of applications (Klemm et al. 2011; Moon et al. 2011; Thomas et al. 2018). CNMs in the form of cellulose nanocrystals (CNCs) or cellulose nanofibrils (CNFs) can be produced by acid hydrolysis or mechanical and chemical defibrillation, respectively

T. Kaldéus · E. Malmström

Department of Fibre and Polymer Technology, School of Engineering Sciences in Chemistry, Biotechnology and Health, KTH Royal Institute of Technology, Teknikringen 56-58, 10044 Stockholm, Sweden

T. Kaldéus · E. Malmström · L. Bergström Wallenberg Wood Science Center, KTH Royal Institute of Technology, Teknikringen 56-58, 10044 Stockholm, Sweden

I. Zozoulenko

Wallenberg Wood Science Center, Linköping University, 60174 Norrköping, Sweden
(Phanthong et al. 2018). Sulfuric acid hydrolysis or the chemical modification (i.e., TEMPO mediated oxidation or carboxymethylation) replaces the primary hydroxyl group $(-\mathrm{OH})$ on cellulose surface by common functional groups such as sulfate $\left(-\mathrm{OSO}_{3}{ }^{-}\right)$, carboxylate $\left(-\mathrm{COO}^{-}\right)$and carboxymethyl $\left(-\mathrm{CH}_{2-}\right.$ $\mathrm{COO}^{-}$) (Saito and Isogai 2004; Liimatainen et al. 2013; Eyley and Thielemans 2014; Candido and Gonçalves 2016; Kaldéus et al. 2018).

CNFs and CNCs have been extensively used to make thin films (Lavoine and Bergström 2017; Kontturi and Spirk 2019), foams and aerogels (Lavoine and Bergström 2017; Kontturi and Spirk 2019) for a wide range of applications including e.g., packaging (Trache et al. 2016), photonics (Shrestha et al. 2017; Chen et al. 2020), thermal insulation (Antonini et al. 2019), pharmaceuticals (Picheth et al. 2017), and membranes ( $\mathrm{Li}$ et al. 2019). CNM-based materials often display excellent mechanical, optical, barrier, and heat insulation properties (Lavoine and Bergström 2017; Hamad 2017; Parker et al. 2018). However, cellulose is naturally hygroscopic, i.e., its properties change drastically when exposed to a humid environment due to the presence of hydroxyl groups on the surface. Indeed, the moisture content in wood, which consists mainly of cellulose, lignin, hemicellulose and extractives, can vary from 10 to $40 \%$ depending on the relative humidity $(\mathrm{RH})$, where $\mathrm{RH}$ at a given temperature is defined as the ratio of partial pressure of vapor to the vapor pressure of water. Interestingly, the moisture uptake depends also on functionalization of cellulose. For example, TEMPOoxidized cellulose nanofibrils adsorb more moisture compared to the sulfated cellulose nanofibrils at a $\mathrm{RH}$ 
of 95\% (Guo et al. 2018). Furthermore, there are numerous studies that have showed that the strength, toughness and Young's modulus of cellulosic films is significantly reduced with increasing RH (Benítez et al. 2013; Benítez and Walther 2017) and that the fiber-matrix adhesion can be reduced by moisture uptake (Haslach 2000; Mokhothu and John 2015). Moisture uptake by packaging materials for the pharmaceutical industry can also cause stability problems for moisture sensitive drugs (Mihranyan et al. 2004). However, the quick and sensitive response of cellulose towards moisture can be used in humidity sensors following the change in gravimetric or viscoelastic properties due to water uptake (Solin et al. 2020). Similarly, humidity-responsive chiral nematic CNC films utilize the increase in the chiral nematic pitch of the helix with uptake of water to the change of color of the film (Chen et al. 2020). Recently, it was also shown that moisture-induced swelling of CNM-based foams can also reduce the thermal conductivity due to enhanced phonon scattering (Apostolopoulou-Kalkavoura et al. 2021).

The water uptake in cellulose is a microscopic phenomenon that can be captured by atomistic modelling. The moisture uptake in cellulose depends on several factors such as the cellulose-cellulose, cellulose-water and cellulose-counterion interactions which are governed by van der Waals and Coulombic interactions. The hydration of cellulose and the bound water inside the cellulose were studied using molecular dynamics (MD) simulation (Garg et al. 2020). However, to investigate the effect of the RH on the moisture uptake inside the porous material the Grand Canonical Monte Carlo (GCMC) simulations are required. This is because the GCMC simulations allow the number of atoms in the system to fluctuate, which is needed to describe the case when water molecules are absorbed. Note that the GCMC simulations possess the drawback of constant volume meaning that the structure of cellulose does not change during the GCMC steps. However, the water uptake in cellulose is accompanied by structural changes and increase in pore volume. This cannot be captured by GCMC simulations alone and thus requires MD simulations as well (Kulasinski et al. 2015; Chen et al. 2018).

To design and fabricate high-performance biopolymer-based materials, the mechanism of moisture uptake must be well understood in order to utilize these materials efficiently in various applications. For instance, the moisture uptake in chitosan films decreases with increasing temperature (Aguirre-Loredo et al. 2017). The presence of surface charge and counterions seems to influence the sorption behavior of cellulose indicating that moisture sorption in sulfated cellulose is higher with $\mathrm{H}^{+}$and $\mathrm{Na}^{+}$counterion as compared to $\mathrm{Ca}^{2+}$ counterion (Berthold et al. 1994). However, even if it is known that crystallinity, surface modification and temperature are crucial parameters affecting the moisture uptake of cellulose and CNMs (Kulasinski et al. 2015; Kulasinski 2016), their effect is not fully explored and remains not well understood.

Here we combined a hybrid Grand Canonical Monte Carlo/Molecular Dynamics simulation (GCMC/MD) with gravimetric moisture uptake measurements to investigate the effect of temperature and the degree of crystallinity on moisture uptake of nanocellulose. The experimental measurements were performed on CNM foams prepared from CNCs and CNFs with different surface modifications and the simulations were performed on amorphous cellulose films and crystalline bundles with corresponding surface modifications. The moisture uptake was calculated at temperatures between 285 and $313 \mathrm{~K}$ and RH between 20 and $80 \%$. The calculated data were correlated to various microscopical properties such as the radial distribution functions and the pore size distribution to understand the microscopic mechanism of moisture uptake.

\section{Materials and methods}

\section{Materials}

A never-dried sulfite dissolving pulp (Domsjö Dissolving Plus) was provided by Domsjö Fabriker AB (Aditya Birla Domsjö, Sweden) and used as starting material for the TEMPO-oxidized cellulose nanofibers and for the preparation of carboxymethylated cellulose nanofibers (CMCNFs). NaClO (Alfa Aesar), 2,2,6,6-tetramethyl-1-piperidinyloxy free radical (TEMPO, $\geq 98 \%$, Alfa Aesar), sodium hydroxide (NaOH, P99.2\%, VWR Chemicals), sodium bromide (NaBr, BioUltra, P99.5\%, Sigma Aldrich) and sodium borohydride (NaBH4, $\geq 98 \%$, Sigma-Aldrich) were used as received. Ethanol (EtOH 96\%), methanol 
Table 1 Characterization of the CNM particles and foams

\begin{tabular}{llllllr}
\hline & $\begin{array}{l}\text { Surface } \\
\text { groups }\end{array}$ & $\begin{array}{l}\text { Surface charge } \\
(\mathrm{mmol} \mathrm{COO}-/ \mathrm{g})\end{array}$ & $\begin{array}{l}\text { Particle diameter } \\
(\mathrm{nm})\end{array}$ & $\begin{array}{l}\text { Aspect } \\
\text { ratio }\end{array}$ & $\begin{array}{l}\text { Crystallinity index } \\
(\mathrm{CI})\end{array}$ & $\begin{array}{l}\text { Foam } \rho_{\text {dry }} \\
(\mathrm{kg} \mathrm{m})^{-3}\end{array}$ \\
\hline 1. TCNFs & $-\mathrm{COO}^{-}$ & $1.60 \pm 0.01$ & $2.3 \pm 0.7$ & $202 \pm 16$ & 51.1 & $5.9 \pm 0.2$ \\
2. TCNCs & $-\mathrm{COO}^{-}$ & $1.60 \pm 0.01$ & $4.5 \pm 1.3$ & $89 \pm 36$ & 67.8 & $7.7 \pm 0.3$ \\
3. $\mathrm{CMCNFs}$ & $-\mathrm{CH}_{2} \mathrm{COO}^{-}$ & $0.65 \pm 0.00$ & $3.0 \pm 0.6$ & N/A & 45.8 & $6.3 \pm 0.2$ \\
\hline
\end{tabular}

(MeOH, HPLC grade), 2-propanol (IPA $\geq 99.5$ ), acetic acid (glacial $\geq 99.8 \%$ ) and $\mathrm{NaOH}$ [puriss $>$ $98 \%$, pellets (anhydrous)] were purchased from Sigma Aldrich. Sodium hydrogen carbonate $\left(\mathrm{NaHCO}_{3}\right.$, analysis grade) was purchased from Merck KGaA, Germany and used as received.

The TEMPO-oxidized cellulose nanofibers (TCNFs) were prepared as previously reported using the TEMPO/NaBr/ $\mathrm{NaClO}$ system with $10 \mathrm{mmol}$ of $\mathrm{NaClO}$ per gram of cellulose (Saito and Isogai 2004). The TEMPO-mediated oxidation was performed at $\mathrm{pH}$ 10 for $4 \mathrm{~h}$. The residual aldehyde and ketone groups in the TEMPO-oxidized cellulose pulp were reduced by adding $0.1 \mathrm{~g}$ of $\mathrm{NaBH}_{4}$ per gram of cellulose to the pulp suspension at $\mathrm{pH} 10$ and allowing it to stir for $3 \mathrm{~h}$ (Saito and Isogai 2006). The TEMPO-oxidized cellulose pulp obtained was washed thoroughly with deionized water (DI) to remove the excess reagents. The final TCNF material was obtained by grinding the TEMPO-oxidized cellulose pulp using a Supermasscolloider grinder (Model MKZA10-15 J, Masuko Sangyo Co., Ltd, Japan) equipped with non-porous grinding stones containing silicon carbide (Disk model MKE), using a gap clearance of $100 \mu \mathrm{m}$ at a motor frequency of $30 \mathrm{~Hz}$.

The TEMPO-oxidized CNCs (TCNCs) were obtained from TCNFs prepared with $10 \mathrm{mmol} / \mathrm{g}$ of $\mathrm{NaClO}$. The TCNCs were obtained by $\mathrm{HCl}(2.5 \mathrm{M})$ acid hydrolysis of the TCNFs for $4 \mathrm{~h}$ at $105^{\circ} \mathrm{C}$ (Tanaka et al. 2015) as previously reported followed by centrifugation at $12000 \mathrm{G}$ for $10 \mathrm{~min}$ and dialysis through a membrane with a molecular weight cut-off of $14000 \mathrm{Da}$ against deionized water for 2 weeks to thoroughly clean the final material.

As mentioned above, the same never-dried sulfite dissolving pulp (Aditya Birla, Domsjö Fabriker $\mathrm{AB}$ ) was also used for the preparation of carboxymethylated cellulose nanofibers (CMCNFs).
Prior to use the pulp was washed with acid ( $\mathrm{HCl}, \mathrm{pH}$ 2), DI-water and base $(\mathrm{NaOH}, \mathrm{pH}$ 9) followed by a thorough wash with DI-water and reslushed at 30,000 revolutions (PTI Austria).

CMCNFs were prepared following procedures previously described (Wågberg et al. 2008; Kaldéus et al. 2018). In short, cellulose fibers ( $3 \mathrm{~g})$ were solvent exchanged to ethanol $(96 \%, 150 \mathrm{~mL}, 15 \mathrm{~min})$ four times, where after the fibers were impregnated for $30 \mathrm{~min}$ in monochloroacetic acid $(0.3 \mathrm{~g})$ in isopropanol $(150 \mathrm{~mL})$. The fibers were then placed in an alkaline alcoholic solution $(0.49 \mathrm{~g} \mathrm{NaOH}, 15 \mathrm{~mL}$ $\mathrm{MeOH}$ and $60 \mathrm{~mL}$ iso- $\mathrm{PrOH}$ ) at $82{ }^{\circ} \mathrm{C}$ under reflux. The carboxymethylation was conducted for $60 \mathrm{~min}$ where after work-up was conducted by washing and filtration steps; DI-water $(1 \mathrm{~L})$, acetic acid $(100 \mathrm{~mL}$, $0.1 \mathrm{M})$, and DI-water $(1 \mathrm{~L})$. To convert the pulp into the $\mathrm{Na}+$ form, the fibers were immersed in NaHCO3solution $(4 \mathrm{wt} \%)$ and post-filtered with DI water $(1 \mathrm{~L})$. Finally, the carboxymethylated pulp was mechanically disintegrated in a high pressure microfluidizer (M-110EH, Microfluidics) by a single pass through the $400 \mu \mathrm{m}$ and $200 \mu \mathrm{m}$ chambers and four times through the $200 \mu \mathrm{m}$ and $100 \mu \mathrm{m}$ chambers. The homogenized fibers were diluted with Milli Q-water $(0.15 \mathrm{wt} \%)$ then ultrasonicated (10 $\mathrm{min}, 30 \%$ amplitude, using microtip probe (6 $\mathrm{mm}$ in diameter)) on a Vibracell VCX750 (Sonics \& Materials Inc., US). Thereafter, the sonicated dispersions were centrifuged for $1 \mathrm{~h}$ at a gravitational force of $4100 \mathrm{~g}$ using a Rotina 420 bench-top centrifuge (Andreas Hettich $\mathrm{GmbH} \& \mathrm{Co.KG}$, Germany) and the supernatant was collected.

Note that the initial pulp was never-dried and its moisture content was $10-15 \%$. After performing the TEMPO oxidation and carboxymethylation followed by mechanical disintegration we obtained TCNF and $\mathrm{CMCNF}$ homogeneous and flowable colloidal 
dispersions with a concentration of $0.5 \mathrm{wt} \%$, respectively. The same applied for the TCNC dispersion which had a concentration around $0.1 \mathrm{wt} \%$ after dialysis and was concentrated up to $0.5 \mathrm{wt} \%$ using a rotary evaporator. The aqueous dispersions were homogenized using an UltraTurrax before freezecasting to ensure the homogeneity of the dispersions.

\section{Preparation of anisotropic CNM foams}

Anisotropic TCNF, TCNC and CMCNF foams were prepared by unidirectional ice templating (Wicklein et al. 2015; Munier et al. 2016) from $0.5 \mathrm{wt} \%$ dispersions $0.5 \mathrm{wt} \%$ in DI. The unidirectional icetemplating was performed using teflon molds of $4 \mathrm{~cm}$ diameter and $2.5 \mathrm{~cm}$ height equipped with copper bottom plate that were filled with TCNF, TCNC or CMCNF dispersion and placed in contact with a dry ice block, giving a final cooling rate of $3 \mathrm{~K} \mathrm{~min}^{-1}$. The final dry foams were obtained by removing the ice by sublimation at 0.024 mbar and room temperature for four days using a freeze-dryer (Christ Alpha 1-2LDplus, Germany). TCNF, TCNC and the CMCNF foams prepared from the same type of starting materials (and same batch) were used in all the experiments.

\section{CNM particle and CNM Foam characterization}

AFM (Dimension 3100, Bruker, USA) operated in tapping mode was used to determine the CNM dimensions (Fig. 1). A droplet of $0.0010 .005 \mathrm{wt} \%$ aqueous CNM dispersion was deposited onto freshly cleaved mica substrate and dried at ambient conditions.

Conductometric titration (Committee 2002) with $\mathrm{NaOH}$ as titrant was used to determine the surface charge of CNMs to be $1.60 \mathrm{mmol} \mathrm{COO}^{-}$per gram of cellulose for the TCNFs and TCNCs, and $0.65 \mathrm{mmol}$ $\mathrm{COO}^{-}$per gram of cellulose for the CMCNFs.

SEM images of the CNM foam cross-section were taken using a HITACHI TM-3000 (Germany) using a $5 \mathrm{kV}$ electron beam at a magnification of $\times 500$ (Fig. 2). The foam samples were coated with gold in argon atmosphere for $20 \mathrm{~s}$.

The apparent density, $\rho$, of the foams was calculated from the mass and the volume (height $\times \pi r^{2}$ ) of the foams, kept for $3 \mathrm{~d}$ at $50 \% \mathrm{RH}$ and $295 \mathrm{~K}$.
The porosity $(\Pi)$ of the foams was determined from the skeletal $\left(\rho_{\text {skel }}\right)$ (Wicklein et al. 2015) and the apparent foam density $\left(\rho_{\text {app }}\right)$.

The crystallinity index (CI) of the CNM foams was calculated from 1D diffractograms obtained with a Panalytical X'Pert PRO diffractometer operated with $\mathrm{Cu} \mathrm{K} \alpha$ radiation and in Bragg-Brentano diffraction geometry. Thin pressed CNM foam samples with a thickness less than $1 \mathrm{~mm}$ that were prepared by axially compressing CNM foams were mounted on a Si wafer zero-background holder spinning at a constant rate of $30 \mathrm{rpm}$ and diffraction patterns were measured for $2 \theta$ between 5 and $50^{\circ}$ with $0.0167^{\circ}$ step size.

The CI was then calculated using the Segal method (Segal et al. 1959) (Eq. 1).

$C I(\%)=\frac{I_{200}-I_{a m}}{I_{200}} \times 100$

where $I_{200}$ and $I_{a m}$ are the intensity values for the (200) peak of crystalline cellulose located at $2 \theta=22-23^{\circ}$ and the minimum in the intensity located at $2 \theta=18-19^{\circ}$ that represent the amorphous content. The angular values were measured with a copper $(\mathrm{Cu})$ $\mathrm{X}$-ray source.

Gravimetric moisture uptake

The moisture uptake of the CNM foams under controlled $\mathrm{RH}$ and temperature was determined gravimetrically by measuring the weight change using a high-precision balance (BP 210 S, Sartorius, Germany) placed inside a humidity chamber as described previously (Apostolopoulou-Kalkavoura et al. 2018). Prior to the measurements, the foams were dried at $313 \mathrm{~K}$ and $20 \% \mathrm{RH}$. The moisture content on dry weight (w) basis, $\mathrm{H}_{2} \mathrm{O}_{\mathrm{w}}$, (i.e., the weight of water divided by the weight of dry cellulose) as a function of $R H(20,35,50,65$ and 80\%) was assessed at 285, 295, 303, 308 and $313 \mathrm{~K}$. Each measurement at a specific temperature and $\mathrm{RH}$ lasted six hours to ensure that steady state was reached, the foam mass was measured every five minutes and the mass of the foam changed less than $1 \%$ during the final hour of equilibration.

Computational model

The amorphous and crystalline cellulose were modeled in the present work where TEMPO-oxidized (TEMPO) cellulose and carboxymethylated (CM) 

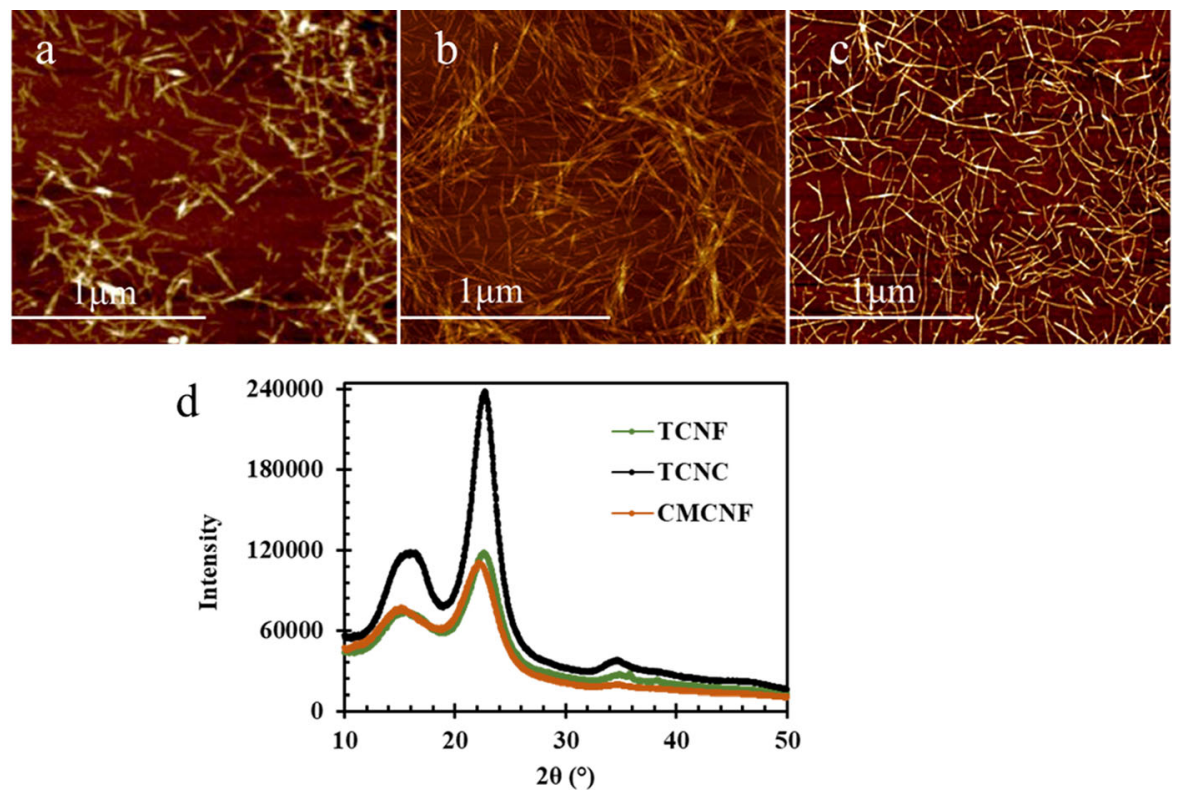

Fig. 1 AFM images of a TCNFs, b TCNCs, and c CMCNFs. d X-ray diffraction spectra for TCNC (black), TCNF (green) and CMCNF (orange) foams used for determining the crystallinity index

cellulose were considered. The cellulose chains with both the functionalities are shown in Figs. 3a, b, where a conventional numbering of carbon atoms (1-6) is indicated. For TEMPO-oxidized cellulose both hydrogen atoms of C6 carbon atom were removed and replaced by one oxygen, and the hydrogen atom of O6 carbon was removed. In case of CM-modification, the hydrogen atom of $\mathrm{O} 6$ oxygen atom was replaced by $\mathrm{CH}_{2} \mathrm{COO}^{-}$group. For both TEMPO and CM-modified cellulose, $\mathrm{Na}^{+}$is used as the counter ion to make the system charge neutral.

The degree of substitution was $1.6 \mathrm{mmol} \mathrm{COO}^{-} / \mathrm{g}$ of cellulose and $0.6 \mathrm{mmol} \mathrm{CH}_{2} \mathrm{COO}^{-} / \mathrm{g}$ of cellulose for TEMPO- and CM-cellulose respectively (both amorphous and nanocrystalline cellulose). For amorphous cellulose, all chains were modified and for crystalline cellulose only surface chains were modified. The number of substituents per glucose residue for TEMPO-amorphous cellulose was 0.256 and for CM-amorphous cellulose was 0.1 . For crystalline cellulose the surface chains only were modified and the number of substituents per glucose residue on the surface is 0.3416 for TEMPO-crystalline cellulose and 0.127 for CM-crystalline cellulose.

The amorphous cellulose chains were generated with Avogadro (Hanwell et al. 2012). The computational box with 16 cellulose chains containing 10 repeating glucose units was generated with an initial density of $1 \mathrm{gm} / \mathrm{cm}^{3}$. To generate the amorphous film, we followed the thermal annealing protocol where the
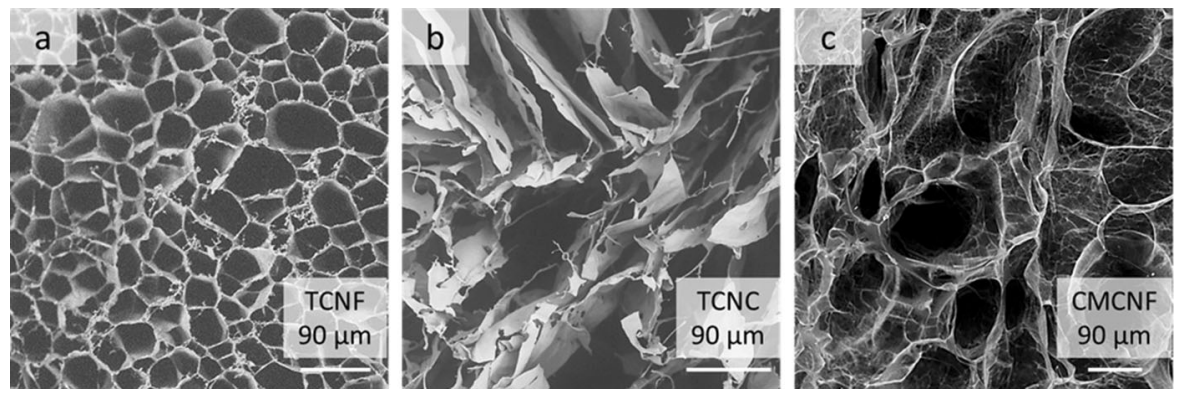

Fig. 2 SEM images of a TCNF, b TCNC, and $\mathbf{c}$ CMCNF foam cross-section 

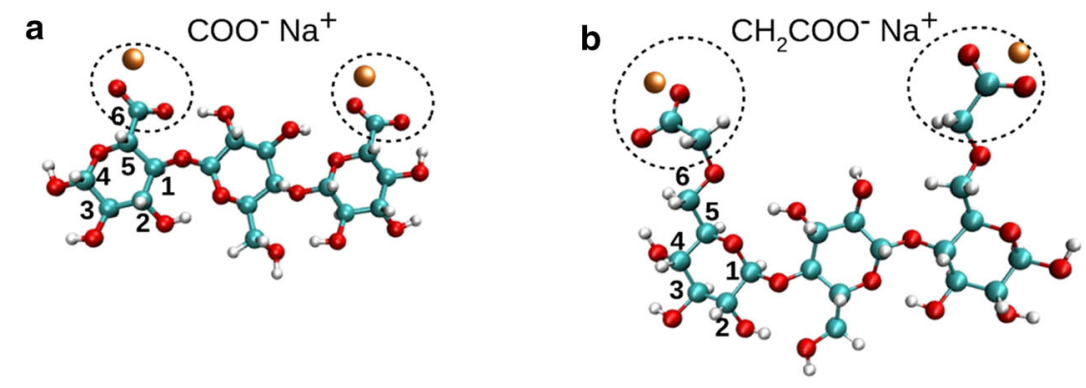

C Amorphous Cellulose

\section{Dry Film}

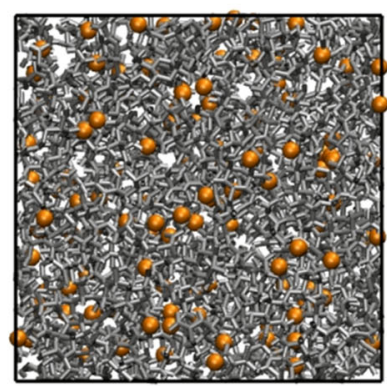

Hydrated Film

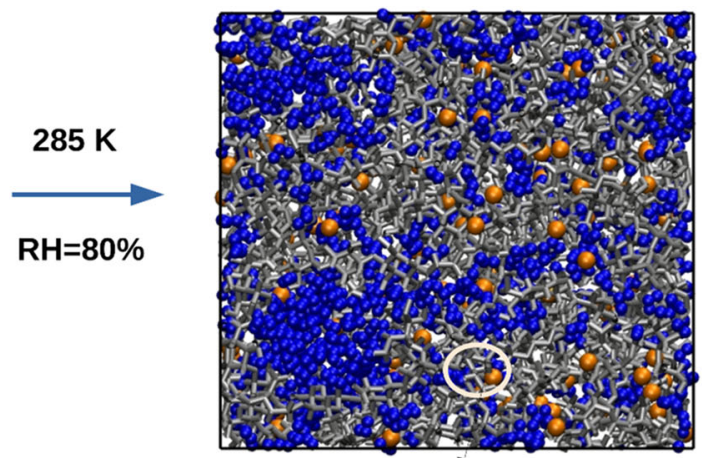

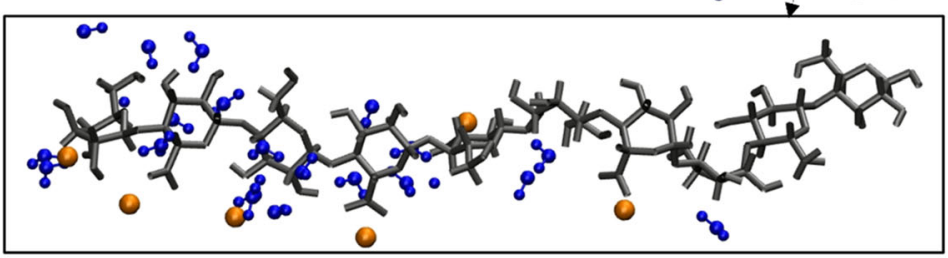

d

Dry Film

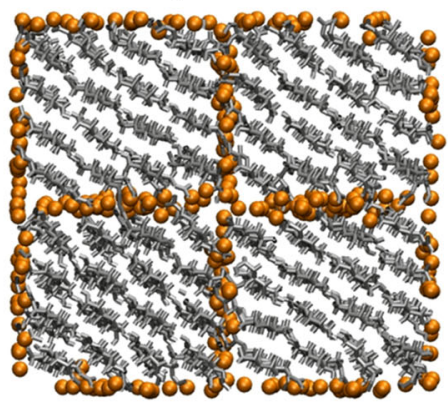

Fig. 3 Cellulose chains functionalized via: a carboxylate groups (TEMPO), b Carboxymethylated (CM) groups and neutralized by $\mathrm{Na}^{+}$counterion (in orange), $\mathbf{c}$ TEMPO-modified amorphous cellulose film at initial dry state and final hydrated state when subjected to $80 \% \mathrm{RH}$ and temperature of $285 \mathrm{~K}$. Insets at the bottom show a representative cellulose chains;
$285 \mathrm{~K}$

\section{$\mathrm{RH}=80 \%$}

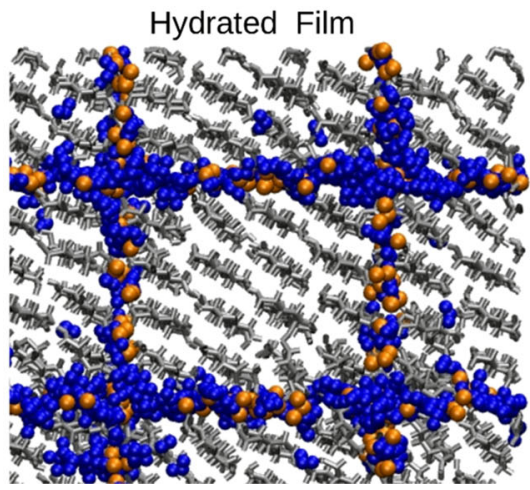

water molecules are depicted in blue. $\mathbf{d}$ Array of four TEMPOmodified cellulose nanocrystals arranged in bundle at initial dry state and hydrated state when subjected to $80 \% \mathrm{RH}$ at $285 \mathrm{~K}$. The cellulose chains and water molecules are shown in grey and blue color respectively 


\section{TEMPO-modified}

a

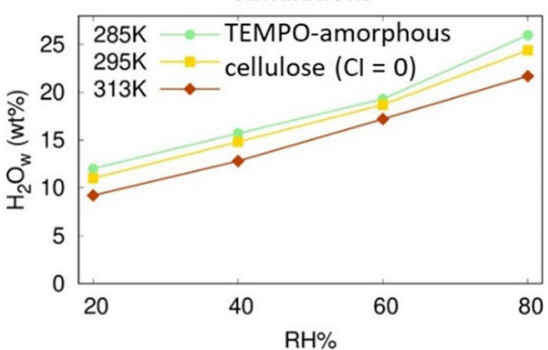

C

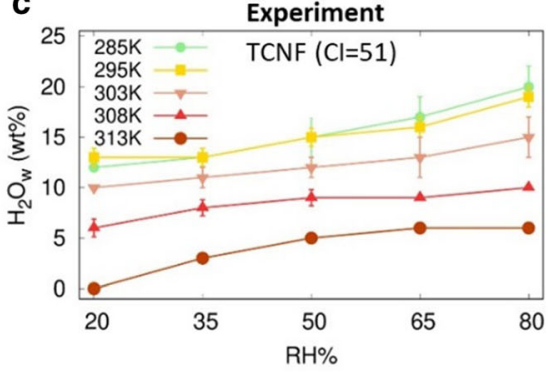

b
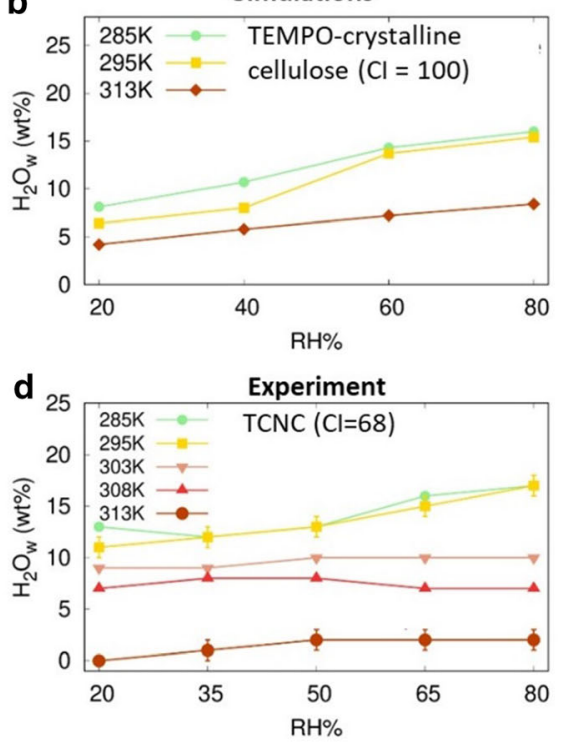

CM-modified
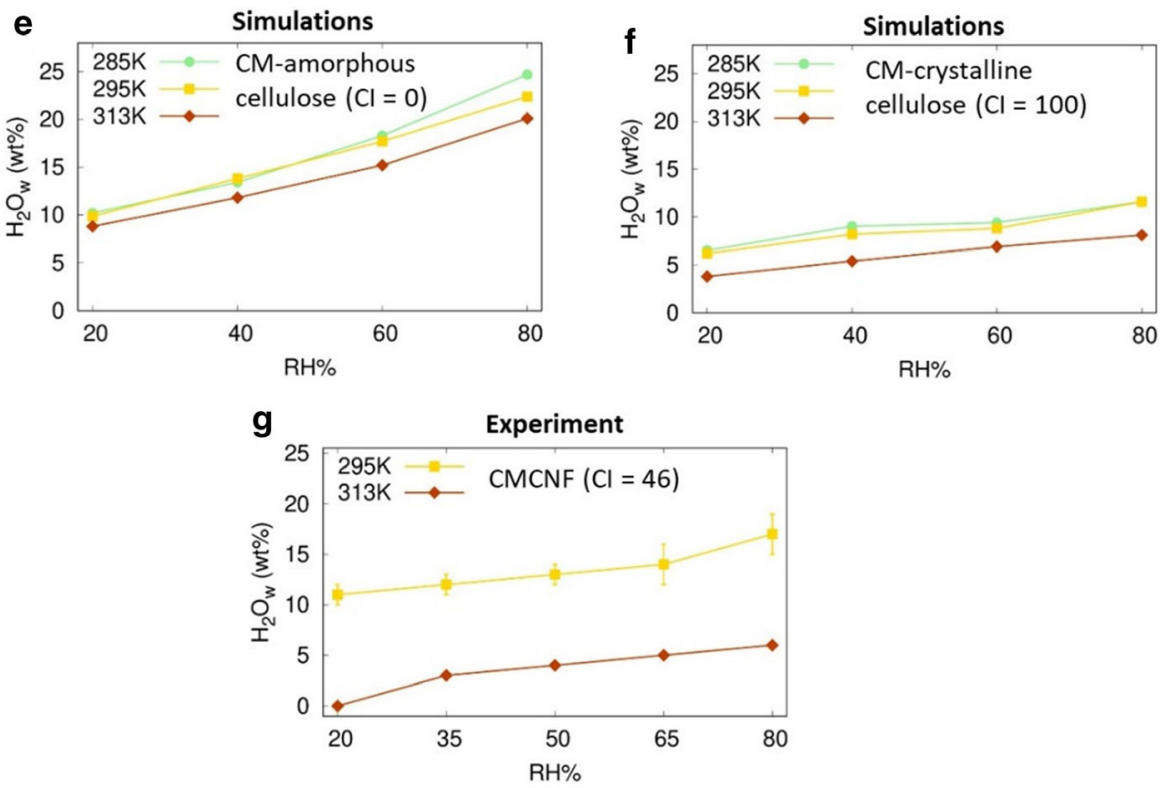

Fig. 4 Simulated and experimental values of moisture uptake (weight percent on dry basis) as a function of relative humidity and at different temperatures for: a TEMPO-modified amorphous cellulose films (simulations); b bundles of TEMPOmodified $100 \%$ crystalline CNCs (simulations); c TCNF foams

film is first heated above the glass transition temperature of polymer and then quenched to the desired temperature. First the cellulose chains were (experimental); d TCNC foams (experimental); e carboxymethylated amorphous cellulose films (simulations); f bundles of carboxymethylated $\mathrm{CNCs}$ (simulations); g CMCNF foams (experimental)

equilibrated at a high temperature of $800 \mathrm{~K}$ in the NPT (constant number of particles, pressure, and temperature) ensemble with Nosé-Hoover thermostat 
TEMPO-amorphous cellulose $(\mathrm{Cl}=0)$
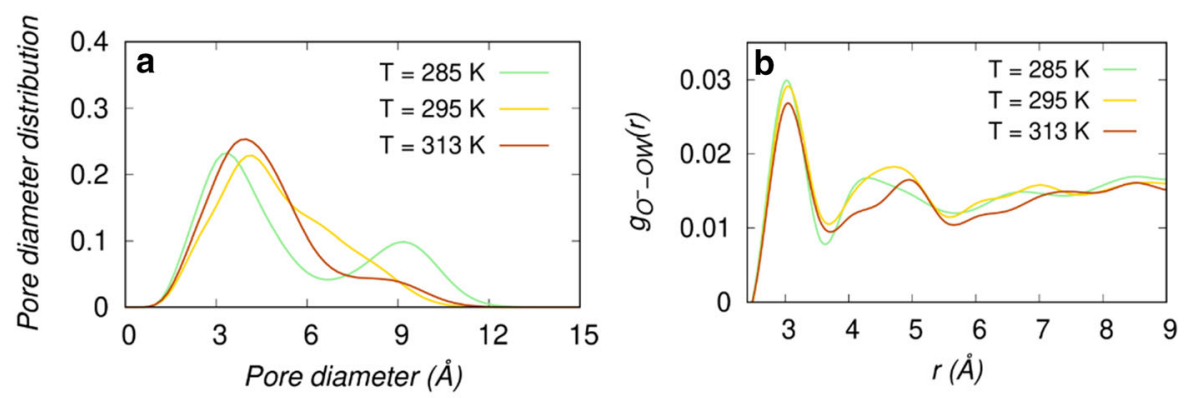

TEMPO-crystalline cellulose $(\mathrm{Cl}=100)$

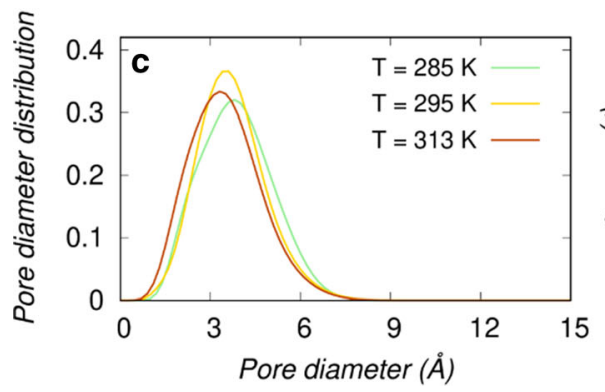

Fig. 5 a and $\mathbf{c}$ Pore diameter distribution as a function of pore diameter, and $\mathbf{b}$ and $\mathbf{c}$ the radial pair distribution $\mathrm{g}_{\mathrm{O}}{ }^{-}$$\mathrm{OW}(\mathrm{r})$ between the oxygen $\left(\mathrm{O}^{-}\right)$of carboxyl group and oxygen (OW) of water for different temperatures $\mathrm{T}=285 \mathrm{~K}, 295 \mathrm{~K}$,

and barostat with a coupling constant of 0.1 and $1 \mathrm{ps}$ respectively. After equilibration at high temperature the amorphous film was quenched from $800 \mathrm{~K}$ to desired temperatures of $285 \mathrm{~K}, 295 \mathrm{~K}$ and $313 \mathrm{~K}$ at a cooling rate of $0.1 \mathrm{~K} / \mathrm{ps}$. The films were then equilibrated at these temperatures. The total time for equilibration was $10 \mathrm{~ns}$ and the final density of the film converged to $1.3 \mathrm{~g} / \mathrm{cm}^{3}$. Figure $3 \mathrm{c}$ and Figure $\mathrm{S} 1 \mathrm{a}$, b show snapshots of amorphous film prepared using the above-mentioned procedure.

The crystalline cellulose consists of a cellulose nanocrystal $(\mathrm{CNC})$ of dimensions $2.3 \times 2.3 \times 11$ $\mathrm{nm}^{3}$. The cellulose chains within the fibril were arranged in a square lattice with $\mathrm{I}_{\beta}$ crystal structure (Nishiyama et al. 2002). Each fibril consists of 16 chains of cellulose with 20 repeating glucose units. Four CNCs were used, and they were arranged in a form of a bundle having a square cross section, see Fig. 3d. Note that the periodic boundary conditions are used, such that this arrangement corresponds to an infinite periodic structure. In order to make a dry film of CNC bundle we run a short NPT simulation

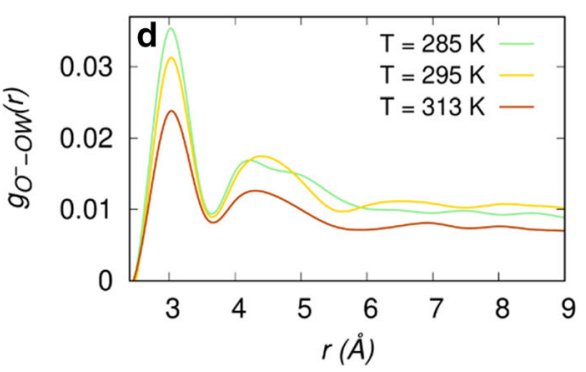

$313 \mathrm{~K}$. Upper and lower panels correspond to respectively TEMPO-amorphous cellulose and TEMPO-crystalline cellulose. The relative humidity is $80 \%$

(constant number of particles, pressure, and temperature) in vacuum to form a bundle. The initial and final configuration of the CNCs in the bundle arrangement is illustrated in Fig. 3d. Figure S1c, d show snapshots of TEMPO-crystalline cellulose and CM crystalline cellulose.

This approach was chosen to create a parallel arrangement of the CNCs with respect to each other as it is done in the experiments.

Note that degree of crystallinity of cellulose can be quantified by the Ramachandran plot showing the distribution of the main dihedral angles about their glycosidic linkage (Khodayari et al. 2021; French et al. 2021). We calculated these distributions for the crystalline and amorphous cellulose for both TEMPOand CM-modified cellulose, see Figure S2.

Simulation details

All simulations were run in LAMMPS molecular dynamics simulation software (Plimpton 1995). The OPLS-AA force field parameters for cellulose was 
used for describing bonded and non-bonded interactions and were generated using the Maestro software suit of Schrödinger software (Schrödinger 2018). The TIP3P water model is used for sorption (Mark and Nilsson 2001). The water molecules were made rigid by using rigid body command in LAMMPS. The Ewald summation method was used for the long-range electrostatic interactions. The moisture uptake was measured by running hybrid Grand Canonical Monte Carlo (GCMC) and Molecular Dynamics (MD) simulations (Plimpton 1995; Chen et al. 2018)). The GCMC simulation allows the insertion/deletion of atoms at a constant volume and a specific chemical potential and MD simulation allows the relaxation of the system at constant stress. In this way the GCMC/ MD approach allows the water intake in a static structure followed by a structural relaxation. The simulations at a specific temperature were performed by running both MD and GCMC steps. Each simulation window consists of running 100 MD steps followed by 1000 GCMC steps. The MD simulations were run in $\mathrm{N} \sigma \mathrm{T}$ (constant number of particles, stress, and temperature) ensemble with Nosé-Hoover thermostat and a constant stress of $0 \mathrm{~Pa}$ was maintained by Nosé-Hoover barostat. The time step for MD simulations was $1 \mathrm{fs}$. This was then followed by GCMC steps. The GCMC simulations were run in $\mu \mathrm{VT}$ (constant chemical potential, volume, and temperature) ensemble, where the water molecules can be adsorbed or desorbed from the cellulose at a specific chemical potential. To equilibrate the system using hybrid GCMC/MD simulations $10^{5}$ windows of coupled GCMC/MD steps were run, which corresponds to a total time of $10 \mathrm{~ns}$ for each simulation.

\section{Pore size calculations}

A size of a pore at any point inside the material is defined as the maximal size of a sphere that can be fitted at that point without an overlap with other atoms. To calculate the pore size first we remove the water from the system. After that pore size inside the cellulose film is calculated using Zeo ++ code (Pinheiro et al. 2013). For all the calculations, the probe diameter of $1 \AA$ was used.

\section{Results and discussions}

Three different types of CNM foams were produced by ice-templating of $0.5 \mathrm{wt} \% \mathrm{TCNF}$, TCNC and CMCNF aqueous suspensions in a mold equipped with a copper bottom and teflon walls to allow unidirectional freezing. The foams were anisotropic with tubular macropores along the freezing direction (Fig. 2). Interestingly, the surface charge density of the TCNF fibrils and the TCNC rods is identical although the different CNMs had quite different crystallinity indexes ( $\mathrm{CI}=51$ and 68 respectively) and aspect ratio (202 and 89, respectively) (Table 1). On the other hand, the CMCNFs possess a lower surface charge density than TCNFs and TCNCs but a similar particle diameter and CI as the TCNFs. The shrinkage after freeze drying resulted in a slightly higher density of the TCNC foams compared to the more robust TCNF foams. The water uptake was determined after conditioning the anisotropic CNM foams for $6 \mathrm{~h}$ at 285, 295, 303, 308 and $313 \mathrm{~K}$ and 20-80\% RH (Apostolopoulou-Kalkavoura et al. 2018).

The effect of RH and temperature on the water uptake was also determined computationally by hybrid GCMC/MD simulations on bundles of $100 \%$ crystalline CNCs (Fig. 2d), and amorphous cellulose films (Fig. 2c) with different surface modifications. The RH depends on the vapor pressure $(P)$ at a specific temperature which in turn is related to the chemical potential. The RH is equal to $P / P_{0}$, with $P_{0}$ being the saturation vapor pressure. The saturation pressure of water at $285 \mathrm{~K}, 295 \mathrm{~K}$ and $313 \mathrm{~K}$ is $1200 \mathrm{~Pa}, 2300 \mathrm{~Pa}$ and $7000 \mathrm{~Pa}$ respectively. At these temperatures, the water vapor is assumed to be ideal gas with chemical potential $(\mu)$ according to Eq. 2.

$\mu-\mu_{0}=k_{B} T \ln \mathrm{RH}$

where $\mu_{0}$ is the chemical potential of water at saturation vapor pressure, $T$ is the temperature and $\mathrm{k}_{\mathrm{B}}$ is the Boltzmann constant. Figure $3 \mathrm{c}$, d present snapshots of the initial and final configurations of both amorphous and crystalline celluloses at $80 \% \mathrm{RH}$ and temperature of $285 \mathrm{~K}$. For the case of the amorphous cellulose (Fig. 3c) the water molecules (blue beads) are distributed in regions of different sizes. For the case of the crystalline cellulose (Fig. 3d) water is 
primarily distributed at the interface between the cellulose nanorods in the bundle, and very little water is sorbed inside the nanocrystal.

The calculated and measured moisture uptake as a function of relative humidity and temperature is shown in Fig. 4 for both TEMPO oxidized and carboxymethylated CNM foams. It should be noted that the simulated materials, amorphous cellulose $(\mathrm{CI}=0)$ and crystalline cellulose $(\mathrm{CI}=100)$ were studied at 0 and $100 \%$ crystallinity, whereas in the experimentally studied samples the crystallinity varied between CI $=46$ and 68 as specified in Table 1 . The simulated and measured dependencies are consistent with each other and show the expected behavior as a function of the RH and temperature as prescribed by Eq. (2). Namely, as the RH increases the moisture uptake in cellulose increases due to the increase of the chemical potential of water according to Eq. (2). At the same time, at a given $\mathrm{RH}$, as the temperature increases the moisture sorption decreases because of the decrease in the chemical potential of water according to Eq. 2. (Note that in Eq. 2, $\ln \mathrm{RH}<0$ ).

The calculated moisture uptake in amorphous cellulose (for a given RH or temperature) is higher as compared to the crystalline cellulose for both TEMPO- and CM-modified surfaces, see Fig. 4a, b, e, f. Note that the calculated moisture uptake curves sometimes show "non-smooth" features, as e.g., a pronounced moisture uptake between 40 and $60 \% \mathrm{RH}$ at $295 \mathrm{~K}$. We relate this behavior to the finite size of our simulated system, and such features are expected to be smoothed out for larger systems. The experimental measurements for TEMPO-modified cellulose show the same trend where the moisture uptake (for a given $\mathrm{RH}$ or temperature) is higher for more amorphous material, i.e., for TCNFs with $\mathrm{CI}=51.1$ as compared to TCNFs with $\mathrm{CI}=67.8$, see Figs. 4c, d. (Note that experimental measurements for CMCNFs are available only for one degree of crystallinity). It is noteworthy that the higher water uptake on less crystalline CNMs corresponds well to previous work by Kulasinski et al. (Kulasinski et al. 2015; Kulasinski 2016). The CMCNF foams exhibit a slightly lower moisture uptake compared to the TCNF foams. However, a direct comparison for carboxymethylated and TEMPO oxidized celluloses cannot be made because of the lower degree of surface modification in the CMCNFs compared to the TCNFs.
Let us now compare quantitatively the experimental and simulated results. The moisture uptake of TCNFs and TCNCs at the relatively low temperatures of $\mathrm{T}=285 \mathrm{~K}$, and $295 \mathrm{~K}$ lies in between the corresponding simulated results for the amorphous and crystalline cellulose (Fig. 4a-d). The same also applies for CMCNFs for the case of $T=295 \mathrm{~K}$ (Fig. 4e-g). This difference is expected because the crystallinity $\mathrm{CI}$ of the experimental measured materials is in between of the simulated amorphous and completely crystalline materials. However, the experimentally determined moisture uptake at high temperatures is lower than the simulated moisture uptake. It should be noted that the moisture uptake at high temperature is significantly lower than at low temperature, which means that small variations in the residual water after preconditioning at $313 \mathrm{~K}$ and $20 \% \mathrm{RH}$ can result in a higher error of the experimentally measured moisture uptake. However, more studies are needed in order to clarify this issue. Moisture uptake on cellulose microfibrils (containing crystalline cellulose and amorphous hemicellulose) estimated by molecular simulations was found to be about $15 \%$ at $80 \% \mathrm{RH}$ at $300 \mathrm{~K}$ (Kulasinski et al. 2015), which is comparable to the moisture uptake in this study. Wood CNF nanopapers, sisal cellulose nanocrystals and sisal microfibrillated cellulose were also found to have a moisture uptake up to $15 \mathrm{wt} \%$ at $80 \% \mathrm{RH}$ and $298 \mathrm{~K}$ (Belbekhouche et al. 2011). The moisture uptake at $298 \mathrm{~K}$ of cellulose powders with different crystallinities was found to range between 10 and $16 \mathrm{wt} \%$ at $80 \%$ $\mathrm{RH}$, and the moisture uptake was found to decrease with increasing crystallinity at $0-80 \%$ RH but the trend was reversed at $\mathrm{RH}>80 \% \mathrm{RH}$. The large water uptake of the highly crystalline Cladophora cellulose was related to the large pore volume (Mihranyan et al. 2004).

To obtain an insight of the moisture uptake on an atomistic level, let us investigate the changes in the morphology of the cellulose when the temperature changes. In what follows, we present results for the case of TEMPO-oxidized cellulose only. CM-modified cellulose shows a similar behavior, and the corresponding results are presented in SI (Figure S3). Figures 5a, c show the calculated pore diameter distribution for different temperatures for respectively TEMPO-amorphous and TEMPO-crystalline cellulose. A comparison of these distributions clearly shows that pores are larger in the amorphous cellulose 
films compared to crystalline bundles. (Note that this can also be seen in the morphology snapshots presented in Figs. 3c, d). In addition, for the case of the crystalline cellulose the pore diameter distribution practically does not change when the temperature is varied. In the amorphous cellulose water can penetrate anywhere, whereas in the crystalline cellulose bundle the preferential adsorption sites are the interfaces between the CNCs. The adsorption of water molecules involves steric repulsions of polymer chains, and the energy penalty to disturb a crystalline rigid structure is higher as compared to disordered amorphous structure. As a result, rigid structure is less porous as compared to the amorphous structure, and less susceptible to formation of new pores. In contrast, for the case of amorphous cellulose (which is less rigid and more structurally flexible), the pore size increases when the temperature decreases (i.e., when the water intake increases). It is noteworthy that for the case of the crystalline cellulose practically all water is absorbed at the interface region where the cellulose nanocrystals are TEMPO-oxidized and held together by counterion ions due to electrostatic interactions, see Fig. 2d. Only less than $<1 \%$ of the absorbed water molecules are able to intercalate between crystalline cellulose sheets. In order to give a more detailed picture of how many water molecules are absorbed at interface, we calculated the ratio of number of water molecules on the surface to the number of surface cellobiose units as a function of RH for TEMPOoxidized crystalline cellulose as shown in Figure S4. Firstly, the water molecules get absorbed due to the hydrophilic nature of both the surface chains as well as counterions. Secondly, number of water molecules increases from two to four as the $\mathrm{RH}$ increases from 20 to $80 \%$. The increase in the number of water molecules is due to the increase in chemical potential of water molecules as a function of $\mathrm{RH}$.

The slopes of the simulated water intake dependencies as a function of $\mathrm{RH}$ are steeper for the amorphous cellulose as compared to crystalline cellulose (Figs. 4a, b and 3e,f), which is directly related to the difference in water-filled pore size and porosity between the two materials.

Figures $5 \mathrm{~b}, \mathrm{~d}$ show the calculated radial distribution functions $\left(g_{\mathrm{O}}{ }^{-}\right.$-Ow $\left.(r)\right)$ between the oxygen $\left(\mathrm{O}^{-}\right)$ of carboxyl group in cellulose and oxygen (OW) of water for respectively TEMPO-amorphous and TEMPO-crystalline cellulose (Note that the distribution functions $\mathrm{g}_{\mathrm{O}^{-} \mathrm{ow}}(\mathrm{r})$ defines the probability to find a water molecule (to be precise, oxygen of the water molecule, $\mathrm{OW}$ ) near oxygen $\left(\mathrm{O}^{-}\right)$belonging to the carboxyl group as a function of distance $r$ ). The distribution functions exhibit a pronounced peak at $r \approx 3 \AA$ signifying a formation of the hydration shells around the oxygen in the carboxyl group. (A weaker peak between 4 and $5 \AA$ corresponds to the second hydration shell). The evolution of $g_{\mathrm{O}}{ }^{-}$-Ow $(r)$ with temperature is strikingly different for the amorphous and crystalline cellulose and is fully consistent with the evolution of the pore size distribution discussed above. Namely, for the case of the amorphous structure the peak practically does not change with temperature (and thus with the water content), which means that the number of water molecules surrounding the oxygen in the carboxyl group remains the same. This is because in this case water molecules fill in additional pores that are formed during water intake. In contrast, no additional pores are created in the case of crystalline cellulose. As a result, the number of absorbed water molecules between the nanocrystals in the bundle increases, which is reflected in the increase of the peak of the distribution function $g_{\mathrm{O}}{ }^{-}$-Ow $(r)$ as the temperature drops (i.e., when water content increases).

Note that above we presented the analysis of the pore diameter evolution with the change of the temperature. We also performed a similar analysis of the case when the RH changes. As discussed above (Fig. 4 and related discussion in the text), the increase of the RH leads to same effect of the increase of the water intake as the decrease of the temperature. Therefore, as expected, the evolution of the pore size distribution and the changes in the distribution function $g_{\mathrm{O}}{ }^{-}$-Ow $(r)$ with the change of $\mathrm{RH}$ exhibit very similar behavior as the one discussed above, see Figure S5.

\section{Conclusions}

In this study, we investigated the moisture uptake in cellulose nanomaterial foams using a combination of the hybrid Grand Canonical Monte Carlo and Molecular Dynamics simulations for TEMPO-oxidized or carboxymethylated amorphous and crystalline cellulose films and experimental measurements on foams made by TEMPO-oxidized cellulose nanofibrils 
(TCNFs) and nanocrystals (TCNCs) and carboxymethylated cellulose nanofibrils (CMCNFs). The TCNFs and TCNCs exhibited the same surface chemistry, which allowed us to investigate the effect of varying crystallinity on the moisture uptake. The CMCNF fibrils having different surface chemistry but similar particle diameter and crystallinity as TCNFs provided useful insight of the surface chemistry effect on the moisture uptake.

Both experiments and simulations showed that the moisture uptake is enhanced when the relative humidity $(\mathrm{RH})$ is increased, or when the temperature is decreased. This behavior is explained by the basic thermodynamics (Eq. (2)) governing the change of the chemical potential of water as a function of the temperature and the RH. Overall, the experimental moisture uptake in all studied materials is in good agreement with the simulations, especially at lower temperatures $(\mathrm{T}=285-295 \mathrm{~K})$. At higher temperatures $(\mathrm{T}=313 \mathrm{~K})$ the moisture uptake in experiments is lower compared to simulations. We attribute this to the presence of the bound water in experimental samples that is difficult to remove. TCNFs and CMCNFs exhibit a similar moisture uptake as a function of temperature and $\mathrm{RH}$, both in experiments and in simulations.

We find that the water uptake in amorphous cellulose (for a given RH or temperature) is higher as compared to the crystalline cellulose for both TEMPO- and CM-modified surfaces. The higher water uptake of amorphous cellulose is related to formation of numerous water-filled large pores.

We hope that the microscopic understanding of the mechanism of water uptake provided in this study will pave the way to prepare high-performance cellulose materials with improved properties for selected applications such as thermal insulation in humid climates or transportation of moisture sensitive goods.

Acknowledgments L B acknowledges support from the Swedish Energy Agency (Energimyndigheten, project 2019-006749), and Formas (project 2015-2032), E M,L B, T $\mathrm{K}$, and I Z acknowledges support from the Wallenberg Wood Science Center (WWSC). I Z acknowledges support of the Swedish Research Council (project 2016-05990), Tröedssons foundation and Åforsk. M L acknowledges support from the Swedish e-Research Centre (SeRC). The computations were performed on resources provided by the Swedish National Infrastructure for Computing (SNIC) at NSC and HPC2N.
Funding Open access funding provided by Linköping University.

\section{Declarations}

Conflict of interest The authors declare that they have no conflict of interest.

Human and animal rights This article does not contain any studies with human participants or animals performed by any of the authors.

Open Access This article is licensed under a Creative Commons Attribution 4.0 International License, which permits use, sharing, adaptation, distribution and reproduction in any medium or format, as long as you give appropriate credit to the original author(s) and the source, provide a link to the Creative Commons licence, and indicate if changes were made. The images or other third party material in this article are included in the article's Creative Commons licence, unless indicated otherwise in a credit line to the material. If material is not included in the article's Creative Commons licence and your intended use is not permitted by statutory regulation or exceeds the permitted use, you will need to obtain permission directly from the copyright holder. To view a copy of this licence, visit http://creativecommons.org/licenses/by/4.0/.

\section{References}

Aguirre-Loredo RY, Rodriguez-Hernandez AI, Velazquez G (2017) Modelling the effect of temperature on the water sorption isotherms of chitosan films. Food Sci Technol 37:112-118. https://doi.org/10.1590/1678-457X.09416

Antonini C, Wu T, Zimmermann T et al (2019) Ultra-porous nanocellulose foams: a facile and scalable fabrication approach. Nanomaterials 9:1-14. https://doi.org/10.3390/ nano9081142

Apostolopoulou-Kalkavoura V, Gordeyeva K, Lavoine N, Bergström L (2018) Thermal conductivity of hygroscopic foams based on cellulose nanofibrils and a nonionic polyoxamer. Cellulose 25:1117-1126. https://doi.org/10.1007/ s10570-017-1633-y

Apostolopoulou-Kalkavoura V, Hu S, Lavoine N et al (2021) Humidity-dependent thermal boundary conductance controls heat transport of super-insulating nanofibrillar foams humidity-dependent thermal boundary conductance controls heat transport of super-insulating nanofibrillar foams. Matter 4:1-14. https://doi.org/10.1016/j.matt.2020.11.007

Belbekhouche S, Bras J, Siqueira G et al (2011) Water sorption behavior and gas barrier properties of cellulose whiskers and microfibrils films. Carbohydr Polym. https://doi.org/ 10.1016/j.carbpol.2010.10.036

Benítez AJ, Torres-Rendon J, Poutanen M, Walther A (2013) Humidity and multiscale structure govern mechanical properties and deformation modes in films of native cellulose nanofibrils. Biomacromol 14:4497-4506. https:// doi.org/10.1021/bm401451m 
Benítez AJ, Walther A (2017) Cellulose nanofibril nanopapers and bioinspired nanocomposites: a review to understand the mechanical property space. J Mater Chem A 5:16003-16024. https://doi.org/10.1039/c7ta02006f

Berthold J, Desbrières J, Rinaudo M, Salmén L (1994) Types of adsorbed water in relation to the ionic groups and their counter-ions for some cellulose derivatives. Polymer (guildf). https://doi.org/10.1016/S0032-3861(05)80048-5

Candido RG, Gonçalves AR (2016) Synthesis of cellulose acetate and carboxymethylcellulose from sugarcane straw. Carbohydr Polym 152:679-686. https://doi.org/10.1016/j. carbpol.2016.07.071

Chen H, Hou A, Zheng C et al (2020) Light- and humidityresponsive chiral nematic photonic crystal films based on cellulose nanocrystals. ACS Appl Mater Interfaces 12:24505-24511. https://doi.org/10.1021/acsami.0c05139

Chen M, Coasne B, Guyer R et al (2018) Role of hydrogen bonding in hysteresis observed in sorption-induced swelling of soft nanoporous polymers. Nat Commun. https:// doi.org/10.1038/s41467-018-05897-9

Cosgrove DJ (2005) Growth of the plant cell wall. Nat Rev Mol Cell Biol 6:850-861. https://doi.org/10.1038/nrm1746

Eyley S, Thielemans W (2014) Surface modification of cellulose nanocrystals. Nanoscale 6:7764-7779. https://doi.org/10. 1039/c4nr01756k

French AD, Montgomery DW, Prevost NT et al (2021) Comparison of cellooligosaccharide conformations in complexes with proteins with energy maps for cellobiose. Carbohydr Polym 264:118004. https://doi.org/10.1016/j. carbpol.2021.118004

Garg M, Linares M, Zozoulenko I (2020) Theoretical rationalization of self-assembly of cellulose nanocrystals: effect of surface modifications and counterions. Biomacromol. https://doi.org/10.1021/acs.biomac.0c00469

Guo X, Liu L, Hu Y, Wu Y (2018) Water vapor sorption properties of TEMPO oxidized and sulfuric acid treated cellulose nanocrystal films. Carbohydr Polym 197:524-530. https://doi.org/10.1016/j.carbpol.2018.06. 027

Hamad WY (2017) Cellulose nanocrystals: properties production and applications. Wiley, Chichester, UK

Hanwell MD, Curtis DE, Lonie DC et al (2012) Avogadro: an advanced semantic chemical editor, visualization, and analysis platform. J Cheminform 4:17. https://doi.org/10. 1186/1758-2946-4-17

Haslach HW (2000) Moisture and rate-dependent mechanical properties of paper: a review. Mech Time-Dependent Mater 4:169-210. https://doi.org/10.1023/A: 1009833415827

Kaldéus T, Larsson PT, Boujemaoui A, Malmström E (2018) One-pot preparation of bi-functional cellulose nanofibrils. Cellulose 25:7031-7042. https://doi.org/10.1007/s10570018-2066-y

Khodayari A, Hirn U, Spirk S et al (2021) Recrystallization and size distribution of dislocated segments in cellulose microfibrils-A molecular dynamics perspective. Cellulose. https://doi.org/10.1007/s10570-021-03906-7

Klemm D, Kramer F, Moritz S et al (2011) Nanocelluloses: a new family of nature-based materials. Angew Chemie-Int Ed 50:5438-5466. https://doi.org/10.1002/anie.201001273
Kontturi E, Spirk S (2019) Ultrathin films of cellulose: a materials perspective. Front Chem 7:1-18. https://doi.org/ 10.3389/fchem.2019.00488

Kulasinski K (2016) Effects of water adsorption in hydrophilic polymers. Polym Sci Res Adv Pract Appl Educ Asp. Formatex Research Center: Badajoz, Spain 217-223

Kulasinski K, Guyer R, Derome D, Carmeliet J (2015) Water adsorption in wood microfibril-hemicellulose system: role of the crystalline-amorphous interface. Biomacromol 16:2972-2978. https://doi.org/10.1021/acs.biomac. 5 b00878

Lavoine N, Bergström L (2017) Nanocellulose-based foams and aerogels: processing, properties, and applications. J Mater Chem A 5:16105-16117. https://doi.org/10.1039/ c7ta02807e

Li T, Li SX, Kong W et al (2019) A nanofluidic ion regulation membrane with aligned cellulose nanofibers. Sci Adv 5:1-7. https://doi.org/10.1126/sciadv.aau4238

Liimatainen H, Visanko M, Sirviö J et al (2013) Sulfonated cellulose nanofibrils obtained from wood pulp through regioselective oxidative bisulfite pre-treatment. Cellulose 20:741-749. https://doi.org/10.1007/s10570-013-9865-y

Mark P, Nilsson L (2001) Structure and dynamics of the TIP3P, SPC, and SPC/E water models at $298 \mathrm{~K}$. J Phys Chem A 105:9954-9960. https://doi.org/10.1021/jp003020w

Mihranyan A, Llagostera AP, Karmhag R et al (2004) Moisture sorption by cellulose powders of varying crystallinity. Int J Pharm 269:433-442. https://doi.org/10.1016/j.ijpharm. 2003.09.030

Mokhothu TH, John MJ (2015) Review on hygroscopic aging of cellulose fibres and their biocomposites. Carbohydr Polym 131:337-354. https://doi.org/10.1016/j.carbpol.2015.06. 027

Moon RJ, Martini A, Nairn J et al (2011) Cellulose nanomaterials review: structure, properties and nanocomposites. Chem Soc Rev 40:3941. https://doi.org/10.1039/ c0cs00108b

Munier P, Gordeyeva K, Bergström L, Fall AB (2016) Directional freezing of nanocellulose dispersions aligns the rodlike particles and produces low-density and robust particle networks. Biomacromol 17:1875-1881. https://doi.org/10. 1021/acs.biomac.6b00304

Nishiyama Y, Langan P, Chanzy H (2002) Crystal structure and hydrogen-bonding system in cellulose $\mathrm{I} \beta$ from synchrotron $\mathrm{X}$-ray and neutron fiber diffraction. J Am Chem Soc 124:9074-9082. https://doi.org/10.1021/ja0257319

Parker RM, Guidetti G, Williams CA et al (2018) The selfassembly of cellulose nanocrystals: hierarchical design of visual appearance. Adv Mater. https://doi.org/10.1002/ adma.201704477

Phanthong P, Reubroycharoen P, Hao X, Xu G (2018) Nanocellulose: extraction and application. Carbon Resour Convers 1:32-43. https://doi.org/10.1016/j.crcon.2018.05. 004

Picheth GF, Pirich CL, Sierakowski MR et al (2017) Bacterial cellulose in biomedical applications: a review. Int $\mathrm{J}$ Biol Macromol 104:97-106. https://doi.org/10.1016/j.ijbiomac. 2017.05.171

Pinheiro M, Martin RL, Rycroft CH et al (2013) Characterization and comparison of pore landscapes in crystalline 
porous materials. J Mol Graph Model 44:208-219. https:// doi.org/10.1016/j.jmgm.2013.05.007

Plimpton S (1995) Fast parallel algorithms for short-Range molecular dynamics. J Comput Phys 117:1-42

Saito T, Isogai A (2004) TEMPO-mediated oxidation of native cellulose. The effect of oxidation conditions on chemical and crystal structures of the water-insoluble fractions. Biomacromol 5:1983-1989. https://doi.org/10.1021/ bm0497769

Saito T, Isogai A (2006) Introduction of aldehyde groups on surfaces of native cellulose fibers by TEMPO-mediated oxidation. Colloids Surf A Physicochem Eng Asp 289:219-225. https://doi.org/10.1016/j.colsurfa.2006.04. 038

Scandinavian Pulp and Paper and Board Testing Committee (2002) SCAN_-Test Methods. Scandinavian Pulp, Paper and Board Testing Committee, Stockholm.

Schrödinger Release. (2018). Schrödinger Release 2018: Maestro. Schrödinger, LLC.

Segal L, Creely JJ, Martin AE, Conrad CM (1959) An Empirical method for estimating the degree of crystallinity of native cellulose using the X-Ray diffractometer. Text Res J 29:786-794. 004051755902901003

Shrestha S, Diaz JA, Ghanbari S, Youngblood JP (2017) Hygroscopic swelling determination of cellulose nanocrystal (CNC) films by polarized light microscopy digital image correlation. Biomacromol 18:1482-1490. https://doi.org/10.1021/acs.biomac.7b00026

Solin K, Borghei M, Sel O et al (2020) Electrically conductive thin films based on nanofibrillated cellulose: interactions with water and applications in humidity sensing. ACS Appl Mater Interfaces. https://doi.org/10.1021/acsami.0c09997

Tanaka R, Saito T, Hondo H, Isogai A (2015) Influence of flexibility and dimensions of nanocelluloses on the flow properties of their aqueous dispersions. Biomacromol 16:2127-2131. https://doi.org/10.1021/acs.biomac. 5 b00539

Thomas B, Raj MC, Athira BK et al (2018) Nanocellulose, a versatile green platform: from biosources to materials and their applications. Chem Rev 118:11575-11625

Trache D, Hussin MH, Hui Chuin CT et al (2016) Microcrystalline cellulose: isolation, characterization and bio-composites application-A review. Int $\mathrm{J}$ Biol Macromol 93:789-804. https://doi.org/10.1016/j.ijbiomac.2016.09. 056

Wågberg L, Decher G, Norgren M et al (2008) The build-up of polyelectrolyte multilayers of microfibrillated cellulose and cationic polyelectrolytes. Langmuir 24:784-795. https://doi.org/10.1021/la702481v

Wicklein B, Kocjan A, Salazar-Alvarez G et al (2015) Thermally insulating and fire-retardant lightweight anisotropic foams based on nanocellulose and graphene oxide. Nat Nanotechnol 10:277-283. https://doi.org/10.1038/nnano. 2014.248

Publisher's Note Springer Nature remains neutral with regard to jurisdictional claims in published maps and institutional affiliations. 